\title{
Superroughening by linear growth equations with spatiotemporally correlated noise
}

\author{
Ning-Ning Pang \\ Department of Physics, National Taiwan Unversity, Taipei, Taiwan, Republic of China \\ Wen-Jer Tzeng \\ Department of Physics, Tamkang University, Tamsui, Taipei, Taiwan, Republic of China
}

(Received 7 December 2003; published 30 July 2004)

\begin{abstract}
We give an extensive study on a class of interfacial superroughening processes with finite lateral system size in $1+1$ dimensions described by linear growth equations with spatiotemporally power-law decaying correlated noise. Since some of these processes have an extremely long relaxation time, we first develop a very efficient method capable of simulating the interface morphology of these growth processes even in very late time. We numerically observe that this class of superrough growth processes indeed gradually develops macroscopic structures with the lateral size comparable to the lateral system size. Through the rigorous analytical study of the equal-time height difference correlation function, the different-time height difference correlation function, and the local width, we explicitly evaluate not only the leading anomalous dynamic scaling term but also all the subleading anomalous dynamic scaling terms which dominate over the ordinary dynamic scaling term. Moreover, the relation between the macroscopic structure formation and anomalous interfacial roughening of the superrough growth processes is analytically investigated in detail.
\end{abstract}

DOI: 10.1103/PhysRevE.70.011105

PACS number(s): 05.40.-a, 05.70.Ln, 68.35.Ct, 89.75.Fb

\section{INTRODUCTION}

The interfacial roughening phenomenon has drawn considerable interest for its generic behaviors widespread in nature [1]. One of the most important physical quantities related to the interfacial roughening phenomena is the global interfacial width $w(L, t)$, which describes the statistical average of the interface height fluctuation relative to the average interface height over the whole system of lateral size $L$ at time $t$. People have observed that the global interfacial widths $w(L, t)$ of the rough interfaces obey the ordinary dynamic scaling ansatz [2]

$$
w(L, t) \sim \begin{cases}t^{\chi / z} & \text { for } \quad t^{1 / z} \ll L, \\ L^{\chi} & \text { for } \quad t^{1 / z} \gg L .\end{cases}
$$

Here, the two independent exponents $\chi$ and $z$ are known as the global roughness exponent and the dynamic exponent, respectively. In contrast to the global interfacial width $w(L, t)$, the local interfacial width $w(l, t)$ describes the statistical average of the interface height fluctuation relative to the average interface height within the local window of lateral size $l(\ll L)$ at time $t$. From the point of the experimental measurements, the local interfacial width $w(l, t)$ is much more accessible than the global interfacial width $w(L, t)$ for both time and economic concerns. Since it is generally believed that the kinetically roughened interfaces are selfaffine, most people only measure $w(l, t)$ in the experiments.

Recently, this assumption of self-affinity is challenged by the observations of the peculiar interfacial features of the "superrough" growth processes (i.e., the global roughness exponent $\chi>1$ ). People have both experimentally [3-5] and numerically [6-8] observed that the local interfacial widths $w(l, t)$ of the superrough growth processes in finite systems of lateral size $L$ obey the anomalous dynamic scaling ansatz

$$
w^{2}(l, t) \sim\left\{\begin{array}{l}
t^{2 \chi / z} \text { for } t^{1 / z} \ll l, \\
l^{2(\chi-\kappa)} t^{2 \kappa / z} \text { for } l \ll t^{1 / z} \ll L, \\
l^{2(\chi-\kappa)} L^{2 \kappa} \text { for } L \ll t^{1 / z} .
\end{array}\right.
$$

In contrast to the ordinary dynamic scaling ansatz, we see that the spatial scaling here $[w(l, t)$ vs $l]$ in the intermediate and late time regime is described by the local roughness exponent $\chi^{\prime}(\equiv \chi-\kappa)$ instead of the global roughness exponent $\chi$. The third nonzero independent exponent $\kappa$, which describes the local orientational instability of the superrough interfaces, is the signature of the anomalous dynamic scaling behavior.

Interestingly, many fluctuating systems in various fields (such as atmospheric variability [9], currency exchange rates [10], pathological heart dynamics [11], and nucleotide concentrations in deoxyribose nucleic acid sequences [12], etc.) all exhibit trends in addition to stochastic noise. Their data profiles bear much resemblance with the interface morphology of the superrough growth processes. However, the values of the scaling exponents reported in various experiments [3-5,9-12] spread over a considerable range. One source of the different exponent values could be the correlated nature of the noise. The presence of correlations in noise can change the scaling exponents and produce a family of continuously changing universality classes (if a universality class is identified by its scaling exponents). Although the origin of the correlations in noise is not understood, it has been successfully applied to explain many recent experiments in related fields such as the growth of diamond films [13], daily temperature fluctuation in Hungary [14], discharge of neurons in motor and parietal areas of the primate 
cortex [15], bursting dynamics of a fiber laser with an injected signal [16], etc. Thus, we are motivated to take an extensive study on the superrough growth processes with spatiotemporally correlated noise. We will undertake a deep investigation on the superrough growth processes with finite lateral system size of $L$ in $1+1$ dimensions described by the following class of linear growth equations with spatiotemporally correlated noise:

$$
\begin{aligned}
\partial_{t} h(x, t)= & (-1)^{m+1} \nu \partial_{x}^{2 m} h(x, t)+\eta(x, t) \\
& \text { with positive integer } m,
\end{aligned}
$$

where $h(x, t)$ denotes the interface height at position $x$ and time $t$ and $\eta(x, t)$ represents Gaussian-distributed noise of zero mean and power-law decaying correlation

$$
\begin{aligned}
\overline{\eta(x, t) \eta\left(x^{\prime}, t^{\prime}\right)}= & D\left|x-x^{\prime}\right|^{2 \rho-1}\left|t-t^{\prime}\right|^{2 \theta-1} \\
& \text { with } 0 \leqslant \rho<1 / 2 \text { and } 0 \leqslant \theta<1 / 2 .
\end{aligned}
$$

Here and throughout this paper, the overbar denotes the statistical average. Note that, for $m=1$ and 2, Eq. (3) with white noise denotes respectively the well-known EdwardsWilkinson equation [17] and the Mullins-Wolf-Villain equation [18]. By using the simple scaling analysis, it is straightforward to obtain the values of the global roughness exponent $\chi=2 m \theta+\rho+(2 m-1) / 2$ and the dynamic exponent $z=2 m$, which is independent of $\rho$ and $\theta$. Thus, the interfacial growth processes described by Eqs. (3) and (4) with $2 m \theta$ $+\rho+(2 m-1) / 2>1$ display superroughening phenomena.

The outline of this paper is as follows. We first analytically obtain the exact form of the interface heights $h(x, t)$ and then numerically simulate the interface morphology in order to get the overall pictorial features of the growth processes described by this class of equations. Then, we will undertake an extensive analytical study of the equal-time height difference correlation function. Special attention will be paid to the intermediate and late time asymptotic behavior of $G(r, t)$, because the anomalous dynamic scaling behaviors of superrough interfaces appear in these two regimes. For completeness, we also derive the different-time height difference correlation function at the steady state in detail. Finally, we will focus on the relations among the equal-time height difference correlation function, the local width, and the global width. In addition, the relation between the macroscopic structure formation and anomalous interfacial roughening of superrough growth processes will be discussed in detail.

\section{INTERFACE CONFIGURATION}

Now let us consider a one-dimensional interface $h(x, t)$ defined on a linear substrate, from $x=0$ to $x=L$, with periodic boundary conditions. By Fourier transforming Eq. (3) into $k$-space, we obtain

$$
\begin{aligned}
\partial_{t} \tilde{h}\left(k_{n}, t\right)= & -\nu k_{n}^{2 m} \widetilde{h}\left(k_{n}, t\right)+\widetilde{\eta}\left(k_{n}, t\right), \\
& \text { where } k_{n} \equiv n(2 \pi / L) \text { with } n=0, \pm 1, \pm 2, \ldots .
\end{aligned}
$$

Under the assumption of the flat initial conditions, the solution is then obtained

$$
\tilde{h}\left(k_{n}, t\right)=e^{-\nu k_{n}^{2 m} t} \int_{0}^{t} d \tau e^{\nu k_{n}^{2 m} \tau} \widetilde{\eta}\left(k_{n}, \tau\right)
$$

with

$$
\overline{\tilde{\eta}\left(k_{n}, \tau\right) \tilde{\eta}\left(k_{m}, \tau^{\prime}\right)}=D_{\rho}\left|k_{n}\right|^{-2 \rho}\left|\tau-\tau^{\prime}\right|^{2 \theta-1} \delta_{n,-m} .
$$

Subsequently, by Fourier transforming this result back to $x$ space, the exact form of the interface height $h(x, t)$ can be obtained. We then perform some simulation to pictorially observe the interface morphology. Note that the dynamic exponent $z=2 m$ and thus this class of the growth processes have extremely long relaxation time as the value of $m$ increases. Here we first develop a very efficient method capable of simulating the interface morphology of these growth processes even in the very late time. By employing Eqs. (6) and (7), we have

$$
\begin{aligned}
\overline{\widetilde{h}\left(k_{n}, t\right) \tilde{h}\left(k_{l}, t\right)} & =\delta_{n,-l} D_{\rho}\left|k_{n}\right|^{-2 \rho} \int_{0}^{t} d \tau e^{-\nu k_{n}^{2 m}(t-\tau)} \\
& \times \int_{0}^{t} d \tau^{\prime} e^{-\nu k_{n}^{2 m}\left(t-\tau^{\prime}\right)}\left|\tau-\tau^{\prime}\right|^{2 \theta-1} \\
= & \delta_{n,-l}\left(\frac{D_{\rho}}{\nu^{1+2 \theta}}\right)\left|k_{n}\right|^{-(2 m+2 \rho+4 m \theta)} \\
& \times \int_{0}^{\nu k_{n}^{2 m} t} d \tau e^{-\tau} \tau^{2 \theta-1}\left[1-e^{-2\left(\nu k_{n}^{2 m}{ }^{2 m}\right)}\right] \\
& \equiv \delta_{n,-l} S\left(k_{n}, t\right) .
\end{aligned}
$$

Since all the $\eta(x, t)$ 's follow the normal distribution, all their linear combinations including all the real and imaginary parts of $\widetilde{\eta}\left(k_{n}, t\right)$ 's and $\widetilde{h}\left(k_{n}, t\right)$ 's follow the normal distribution.

We then take the following four steps.

(1) $\widetilde{h}\left(k_{0}, t\right)$ is set to 0 , since it is irrelevant to the relative heights.

(2) $\widetilde{h}\left(k_{L / 2}, t\right)$ is real and follows the normal distribution with mean zero and standard deviation $\sqrt{S\left(k_{L / 2}, t\right)}$.

(3) For $0<n<L / 2, \quad \widetilde{\widetilde{h}\left(k_{n}, t\right) \widetilde{h}\left(k_{-n}, t\right)}=\widetilde{h}\left(k_{n}, t\right) \widetilde{h}\left(k_{n}, t\right)^{*}$ $=S\left(k_{n}, t\right)$ and $\overline{\widetilde{h}\left(k_{n}, t\right) \tilde{h}\left(k_{n}, t\right)}=0$. Thus, $\widetilde{h}\left(k_{n}, t\right)$ is a complex random variable with the real part and the imaginary part mutually independent and both following the normal distribution with zero mean and standard deviation $\sqrt{S\left(k_{n}, t\right) / 2}$.

(4) Perform Fourier transformation of $\left\{\tilde{h}\left(k_{n}, t\right)^{\prime}\right.$ 's $\}$ to get $\left\{h(x, t)^{\prime}\right.$ s $\}$.

We pictorially observe that the growth process described by Eqs. (3) and (4) with $2 m \theta+\rho+(2 m-1) / 2>1$, starting from the flat initial condition, gradually develops a macro- 


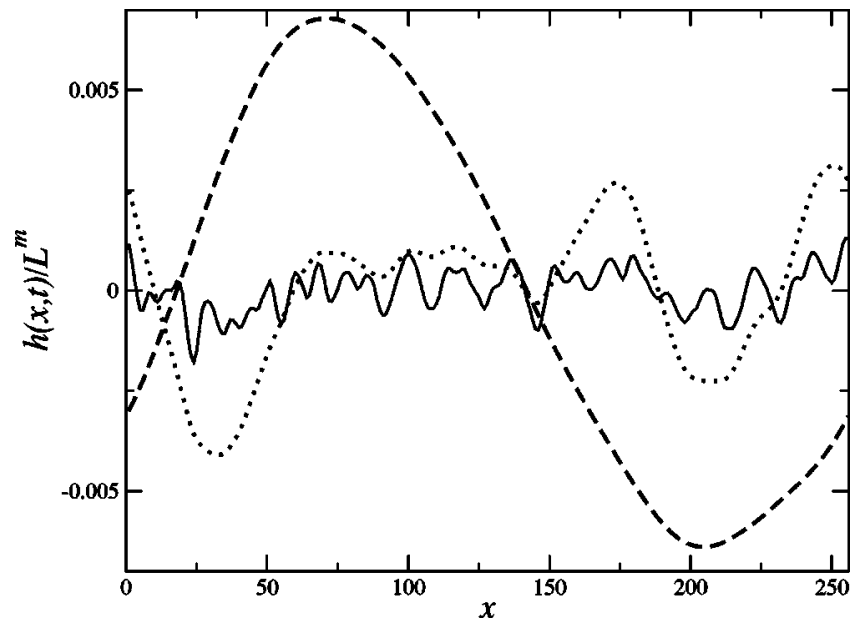

FIG. 1. A series of different-time snapshots of the interface configuration for the growth processes described by Eqs. (3) and (4) with $m=3, \rho=0.2, \theta=0, \nu=1 / 2, D_{\rho}=1$, and the lateral system size $L=256$. The interface configurations at time $t=10, t=10^{5}$, and $t$ $=10^{9}$ are represented by the solid, dotted, and dashed curves and magnified for clarity in $h$ direction by 400 times, 20 times, and unchanged, respectively.

scopic structure with the lateral size comparable to $L$. Besides, the periodic boundary condition restricts the macroscopic structure in the form of global mountains or valleys. For a larger value of $m$, there is less restriction on the local interfacial slope variation and, consequently, the macroscopic structure becomes rougher. Thus, as the value of $m$ increases, the values of the exponents $\chi$ and $\kappa$ both become larger. For illustration, Fig. 1 demonstrates a series of different time snapshots of interface configurations of the growth processes described by Eqs. (3) and (4) with $\rho=0.2$ and $\theta$ $=0$ for $m=3$.

\section{EQUAL-TIME HEIGHT DIFFERENCE CORRELATION FUNCTION}

We will take an extensive analytical study of the equaltime height difference correlation function $G(r, t)$ and rigorously evaluate its asymptotic forms in different time regimes. $G(r, t)$ is defined as

$$
\begin{aligned}
G(r, t) \equiv & \overline{\left\langle[h(x, t)-h(x+r, t)]^{2}\right\rangle_{L}}=\overline{\left\langle h^{2}(x, t)\right\rangle_{L}}+\overline{\left\langle h^{2}(x+r, t)\right\rangle_{L}} \\
& -2 \overline{\langle h(x, t) h(x+r, t)\rangle_{L}}
\end{aligned}
$$

with $\langle\ldots\rangle_{L}$ denoting the lateral spatial average over the whole system of lateral size $L$ throughout this paper. By doing some calculation, we get

$$
\begin{aligned}
\overline{\langle h(x, t) h(x+r, t)\rangle_{L}}= & \frac{2 D_{\rho}}{L} \sum_{n=1}^{\infty} \frac{e^{-i k_{n} r}}{\nu k_{n}^{2 m+2 \rho}} \\
& \times \int_{0}^{t} d \tau\left[e^{-\nu k_{n}^{2 m} \tau}-e^{-\nu k_{n}^{2 m}(2 t-\tau)}\right] \tau^{2 \theta-1} .
\end{aligned}
$$

Subsequently, $G(r, t)$ is obtained as follows:

$$
\begin{aligned}
G(r, t)= & \frac{4 D_{\rho}}{L} \sum_{n=1}^{\infty} \frac{1-\cos \left(k_{n} r\right)}{\nu k_{n}^{2 m+2 \rho}} \int_{0}^{t} d \tau\left[e^{-\nu k_{n}^{2 m} \tau}\right. \\
& \left.-e^{-\nu k_{n}^{2 m}(2 t-\tau)}\right] \tau^{2 \theta-1} .
\end{aligned}
$$

It is easily seen from Eq. (11) that there exists a characteristic wave vector $k_{c}\left[\equiv(\nu t)^{-1 / 2 m}\right]$. It separates the time evolution of $G(r, t)$ into three regimes: $k_{c} \gg 1 / r, 1 / r \gg k_{c} \gg 1 / L$, and $k_{c} \ll 1 / L$.

For $k_{c} \gg 1 / L$, we can approximate Eq. (11) by taking the limit $L \rightarrow \infty$ and thus

$$
\begin{aligned}
\left.G(r, t)\right|_{k_{c} \gg 1 / L} \simeq & \frac{2 D_{\rho}}{\pi \nu} \int_{0}^{\infty} d k \frac{1-\cos (k r)}{k^{2 m+2 \rho}} \\
& \times \int_{0}^{t} d \tau\left[e^{-\nu k^{2 m} \tau}-e^{-\nu k^{2 m}(2 t-\tau)}\right] \tau^{2 \theta-1} \\
= & \frac{2 D_{\rho}}{\pi \nu^{1+2 \theta} k_{c}^{2 \rho+4 m \theta+2 m-1}} \int_{0}^{\infty} d \tau \tau^{2 \theta-1} \\
& \times \int_{\tau^{1 / 2 m}}^{\infty} d x \frac{1-\cos \left(k_{c} r x\right)}{x^{2 \rho+4 m \theta+2 m}}\left(e^{-\tau}-e^{-2 x^{2 m}+\tau}\right) .
\end{aligned}
$$

We then derive the early and intermediate time asymptotes of $G(r, t)$ from Eq. (12). First, for the early time regime $\left(k_{c}\right.$ $\gg 1 / r \gg 1 / L)$, the asymptotes of $G(r, t)$ is readily obtained to the leading order

$$
\left.G(r, t)\right|_{k_{c} \gg 1 / r} \sim O\left(\frac{D_{\rho}}{\nu^{1+2 \theta}} k_{c}^{-(2 \rho+4 m \theta+2 m-1)}\right) .
$$

After substituting $k_{c}=(\nu t)^{-1 / 2 m}$, the global roughness exponent $\chi=2 m \theta+\rho+(2 m-1) / 2$, and the dynamic exponent $z$ $=2 m$ into Eq. (13), the early time asymptote of $G(r, t)$ can also be expressed as

$$
\left.G(r, t)\right|_{t \ll r^{z} / \nu} \sim O\left(\frac{D_{\rho}}{\nu^{(1-2 \rho) / z}} t^{2 \chi / z}\right) .
$$

Next, for the intermediate time regime $\left(1 / L \ll k_{c} \ll 1 / r\right)$, we first employ the following relations

$$
\begin{gathered}
\int_{0}^{\infty} \tau^{2 \theta-1} e^{\tau} d \tau \int_{\tau^{1 / 2 m}}^{\infty} d x f(x) e^{-2 x^{2 m}} \\
=\sum_{j=0}^{\infty} \frac{1}{j !(j+2 \theta)} \int_{0}^{\infty} f(x) x^{2 m j+4 m \theta} e^{-2 x^{2 m}} d x, \\
\int_{0}^{\infty} \tau^{\theta \theta-1} e^{-\tau} d \tau \int_{\tau^{1 / 2 m}}^{\infty} d x f(x)=\int_{0}^{\infty} f(x) \gamma\left(2 \theta ; x^{2 m}\right) d x
\end{gathered}
$$

with $\gamma\left(2 \theta ; x^{2 m}\right)$ denoting the incomplete Gamma function 
[19], then perform the integration by parts systematically, and thus rewrite Eq. (12) as

$$
\begin{aligned}
\left.G(r, t)\right|_{k_{c} \gg 1 / L} \simeq & \frac{2 D_{\rho}}{\pi \nu^{1+2 \theta} k_{c}^{2 \rho+4 m \theta+2 m-1}}\left(\sum _ { j = 0 } ^ { \infty } \frac { 1 } { j ! ( j + 2 \theta ) } \int _ { 0 } ^ { \infty } x ^ { 2 m j + 4 m \theta } d x \left\{\frac{4 m\left(1-\cos k_{c} r x\right)}{2 \rho+4 m \theta+2 m-1} x^{-2 \rho-4 m \theta} e^{-2 x^{2 m}}\right.\right. \\
& -\sum_{p=1}^{m-1}(-1)^{p}\left[\frac{4 m \Gamma(2 m+2 \rho+4 m \theta-2 p)}{\Gamma(2 m+2 \rho+4 m \theta)}\left(k_{c} r\right)^{2 p-1} x^{2 p-1-2 \rho-4 m \theta} e^{-2 x^{2 m}} \sin k_{c} r x\right. \\
& \left.+\frac{4 m \Gamma(2 m+2 \rho+4 m \theta-2 p-1)}{\Gamma(2 m+2 \rho+4 m \theta)}\left(k_{c} r\right)^{2 p} x^{2 p-2 \rho-4 m \theta} e^{-2 x^{2 m}} \cos k_{c} r x\right] \\
& \left.+(-1)^{m} \frac{\Gamma(1+2 \rho+4 m \theta)}{\Gamma(2 m+2 \rho+4 m \theta)}\left(k_{c} r\right)^{2 m-1} x^{-1-2 \rho-4 m \theta} e^{-2 x^{2 m}} \sin k_{c} r x\right\} \\
& \left.-(-1)^{m} \frac{\Gamma(1+2 \rho+4 m \theta)}{2 m \Gamma(2 m+2 \rho+4 m \theta)}\left(k_{c} r\right)^{2 m-1} \int_{0}^{\infty} d y y^{-1-2 \theta-\rho / m} \gamma(2 \theta ; y) \sin \left(k_{c} r y^{1 / 2 m}\right)\right) .
\end{aligned}
$$

Subsequently, by employing the following relations [19]:

$$
\begin{gathered}
\int_{0}^{\infty} x^{\alpha} e^{-2 x^{2 m}} d x=\frac{2^{-(\alpha+1) / 2 m}}{2 m} \Gamma\left(\frac{\alpha+1}{2 m}\right), \\
\int_{0}^{\infty} x^{\alpha-1} \sin \left(b x^{r}\right) \gamma(\nu ; c x) d x=-\frac{b}{c^{\alpha+r}} \sum_{j=0}^{\infty} \frac{\Gamma(\alpha+\nu+r+2 r j)}{(2+r+2 r j)(2 j+1) !}\left(-\frac{b^{2}}{c^{2 r}}\right)^{j}+\frac{\Gamma(\nu) \Gamma(\alpha / r)}{b^{\alpha / r} r} \cos \left(\frac{r-\alpha}{2 r} \pi\right) \\
\text { with }[b, r, \operatorname{Re} c>0 ; \operatorname{Re}(\alpha+\nu)>-r ; \operatorname{Re} \nu>0 ; \operatorname{Re} \alpha<r],
\end{gathered}
$$

we eventually obtain, after some tedious calculation, the intermediate time asymptote of $G(r, t)$ as follows:

$$
\left.G(r, t)\right|_{r^{z} / \nu \ll t \ll L^{z} / \nu} \simeq \frac{2 D_{\rho}}{\pi \nu^{1+2 \theta}} k_{c}^{1-2 m-4 m \theta-2 \rho}\left[\sum_{q=1}^{\infty} C_{2 q}\left(k_{c} r\right)^{2 q}+C\left(k_{c} r\right)^{2 m-1+4 m \theta+2 \rho}\right]
$$

with the coefficients

$$
C_{2 q}=\frac{(-1)^{q+1} 2^{1+2 \theta-(2 q-2 \rho+1) / 2 m}}{(2 m+2 \rho+4 m \theta-2 q-1)(2 q) !} \Gamma\left(\frac{2 q-2 \rho+1}{2 m}\right) B_{1 / 2}\left(2 \theta, 1-\frac{2 q-2 \rho+1}{2 m}\right)
$$

for $q<m$,

$$
\begin{aligned}
C_{2 q}= & \frac{(-1)^{q} \Gamma(1+2 \rho+4 m \theta) \Gamma\left(\frac{2 q-2 \rho+1}{2 m}-1\right)}{2 m \Gamma(2 m+2 \rho+4 m \theta)\left(\frac{2 q-2 \rho+1}{2 m}-1-2 \theta\right)(2 q-2 m+1) !}+\frac{(-1)^{q+1} 2^{1+2 \theta-(2 q-2 \rho+1) / 2 m}}{(2 m+2 \rho+4 m \theta-2 q-1)} \Gamma\left(\frac{2 q-2 \rho+1}{2 m}\right) \\
& \times B_{1 / 2}\left(2 \theta, 1-\frac{2 q-2 \rho+1}{2 m}\right)\left[\frac{1}{(2 q) !}-\frac{\Gamma(1+2 \rho+4 m \theta)}{\Gamma(2 m+2 \rho+4 m \theta)(2 q-2 m+1) !}\right]+\frac{(-1)^{q} 2^{2 \theta-(2 q-2 \rho+1) / 2 m}}{m(2 q-2 m+1) !} \\
& \times \frac{\Gamma(1+2 \rho+4 m \theta) \Gamma\left(\frac{2 q-2 \rho+1}{2 m}-1\right)}{\Gamma(2 m+2 \rho+4 m \theta)} B_{1 / 2}\left(2 \theta, 2-\frac{2 q-2 \rho+1}{2 m}\right)
\end{aligned}
$$


for $q \geqslant m$, and

$$
\begin{aligned}
C= & (-1)^{m} \frac{\Gamma(1+2 \rho+4 m \theta)}{\Gamma(2 m+2 \rho+4 m \theta)} \Gamma(2 \theta) \Gamma(-2 \rho-4 m \theta) \\
& \times \sin (2 m \theta+\rho) \pi
\end{aligned}
$$

with $B_{x}(a, b)$ denoting the incomplete beta function [19]. After substituting $k_{c}=(\nu t)^{-1 / 2 m}$, the global roughness exponent $\chi=2 m \theta+\rho+(2 m-1) / 2$, and the dynamic exponent $z=2 m$ into Eq. (16), the intermediate time asymptote of $G(r, t)$ can also be expressed as

$$
\left.G(r, t)\right|_{r^{z} / \nu \ll t \ll L / \nu} \simeq \sum_{q=1}^{\infty} C_{2 q}^{\prime} t^{2(\chi-q) / z} r^{2 q}+C^{\prime} r^{2 \chi}
$$

with the coefficients $C_{2 q}^{\prime}=C_{2 q}\left[2 D_{\rho} \nu^{2(\chi-q) / z} /\left(\pi \nu^{1+2 \theta}\right)\right]$ and $C^{\prime}=C\left[2 D_{\rho} /\left(\pi \nu^{1+2 \theta}\right)\right]$. Note that, in the right-hand side (rhs) of Eq. (20), the terms $C_{2 q}^{\prime} t^{2(\chi-q) / z} r^{2 q}$ with $q<\chi$ are all dominant over the term $C^{\prime} r^{2 \chi}$ in the limit of $t \gg r^{2} / \nu$. Recall that, for the ordinary dynamic scaling behaviors, the intermediate time asymptote of $G(r, t)$ scales as $r^{2} \chi$; i.e., $G\left(r, t \gg r^{z} / \nu\right)$ $\sim O\left(r^{2} \chi\right)$. Thus, the growth processes described by Eqs. (3) and (4) with $2 m \theta+\rho+(2 m-1) / 2>1$ all display the anomalous dynamic scaling behaviors with the leading anomalous term $C_{2}^{\prime} t^{2(x-1) / z} r^{2}$ and the subleading anomalous terms $\left\{C_{4}^{\prime} t^{2(\chi-2) / z} r^{4}, \ldots, C_{2[\chi]}^{\prime} t^{2(\chi-[\chi]) / z} r^{2[\chi]}\right\}$ dominant over the ordinary dynamic scaling term $C^{\prime} r^{2 \chi}$, where $[\chi]$ denotes the integer part of $\chi$ throughout this paper.

Subsequently, for the late time regime $t \gg L^{z} / \nu$, we now have $k_{c} \ll 1 / L$. The property of $k_{c} \ll 1 / L$ in this regime implies that $\nu k_{n}^{2 m} t \gg 1$ for all positive integer $n$ in Eq. (11):

$$
\begin{aligned}
G(r, t)_{t \gg L^{z} / \nu} \simeq & \frac{4 D_{\rho} \Gamma(2 \theta)}{\nu^{1+2 \theta} L} \sum_{n=1}^{\infty}\left[\frac{1-\cos \left(k_{n} r\right)}{k_{n}^{2 m+2 \rho+4 m \theta}}\right]=\frac{D_{\rho} \Gamma(2 \theta) L^{2 m+2 \rho+4 m \theta-1}}{\nu^{1+2 \theta} \Gamma(2 m+2 \rho+4 m \theta) \cos [\pi(m+\rho+2 m \theta)]}[\zeta(1-2 m-2 \rho-4 m \theta ; 1) \\
& \left.+\zeta(1-2 m-2 \rho-4 m \theta ; 0)-\zeta\left(1-2 m-2 \rho-4 m \theta ; 1-\frac{r}{L}\right)-\zeta\left(1-2 m-2 \rho-4 m \theta ; \frac{r}{L}\right)\right]
\end{aligned}
$$

with $\zeta(\nu ; u)$ denoting the generalized zeta function [19]. By employing the following relations: $\zeta(\nu ; u+1)=\zeta(\nu ; u)-1 / u^{\nu}$ for $u \geqslant 0, \zeta(\nu ; 1)=\zeta(\nu)$ (Riemann's zeta function), and $\partial_{u} \zeta(\nu ; u)=-\nu \zeta(\nu+1 ; u)$, we then obtain

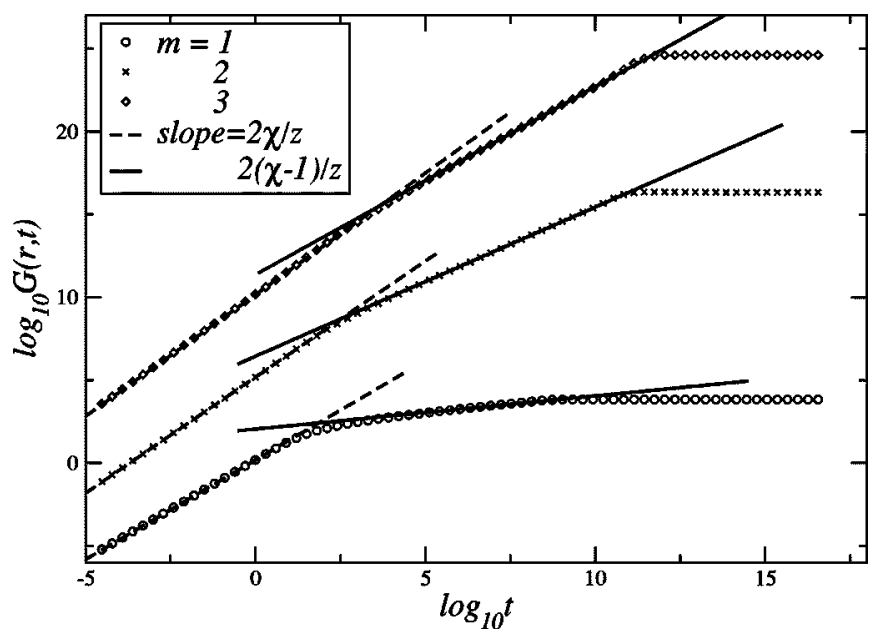

FIG. 2. The log-log plot of the numerical solution of the equaltime height difference correlation function $G(r, t)$ vs time $t$ for the growth processes described by Eqs. (3) and (4) with $m=1$ (circle), $m=2$ (cross), and $m=3$ (diamond), respectively. The data points for the $m=2$ and $m=3$ cases are shifted upward by 5 units and 10 units for visibility.

$$
\begin{gathered}
\zeta(1-\beta ; 1)+\zeta(1-\beta ; 0)-\zeta(1-\beta ; 1-\delta)-\zeta(1-\beta ; \delta) \\
=\delta^{\beta-1}-2 \sum_{p=1}^{\infty} \frac{\delta^{2 p}}{(2 p) !} \frac{\Gamma(2 p+1-\beta)}{\Gamma(1-\beta)} \zeta(2 p+1-\beta) .
\end{gathered}
$$

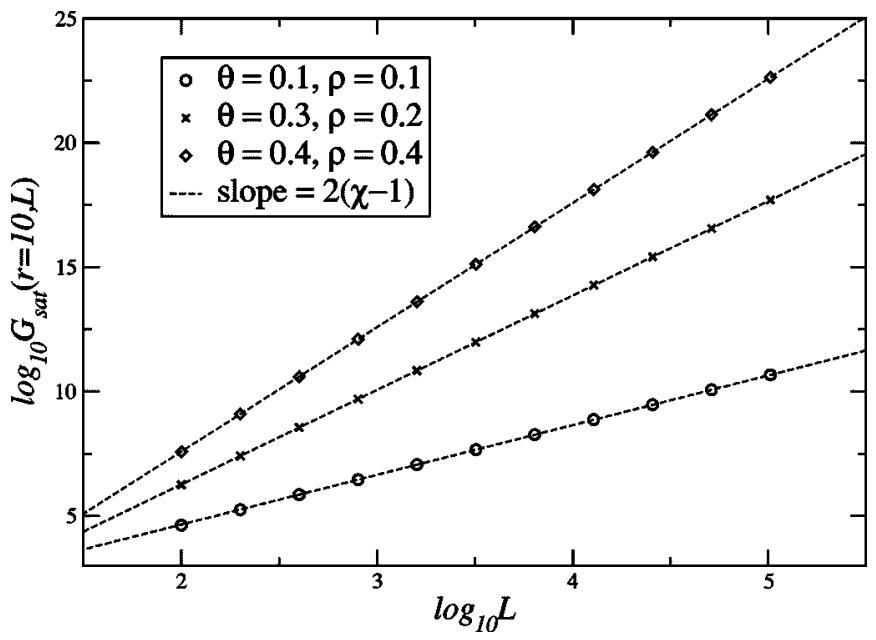

FIG. 3. The log-log plot of the numerical solution of the saturated equal-time height difference correlation function $G_{\text {sat }}(r$ $=10, L)$ vs the lateral system size $L$ for the growth processes described by Eqs. (3) and (4) with $m=2$ and the noise correlation indices $(\rho, \theta)=(0.1,0.1)$ (circle), $(0.2,0.3)$ (cross), and $(0.4,0.4)$ (diamond), respectively. 
Consequently, the late time asymptote of $G(r, t)$ is obtained:

$$
\begin{aligned}
\left.G(r, t)\right|_{t \gg L^{z / \nu}} \simeq & \frac{D_{\rho} \Gamma(2 \theta) L^{2 m+2 \rho+4 m \theta-1}}{\nu^{1+2 \theta}}\left[\sum_{q=1}^{\infty} A_{2 q}(r / L)^{2 q}\right. \\
& \left.+A(r / L)^{4 m \theta+2 \rho+2 m-1}\right]
\end{aligned}
$$

with the coefficients $A_{2 q}=-2 \tan [\pi(m+\rho+2 m \theta)] \Gamma(2 q+1$ $-2 m-2 \rho-4 m \theta) \zeta(2 q+1-2 m-2 \rho-4 m \theta) /[\pi(2 q) !]$ and $A$ $=1 /\{\Gamma(2 m+2 \rho+4 m \theta) \cos [\pi(m+\rho+2 m \theta)]\}$. By substituting the exponent $\chi=2 m \theta+\rho+(2 m-1) / 2$ into Eq. (22), the asymptote of $G(r, t)$ in the late time regime can also be expressed as

$$
\left.G(r, t)\right|_{t \gg L^{z / \nu}} \simeq \sum_{q=1}^{\infty} A_{2 q}^{\prime} L^{2(\chi-q)} r^{2 q}+A^{\prime} r^{2 \chi}
$$

with the coefficients $A_{2 q}^{\prime}=D_{\rho} \Gamma(2 \theta) A_{2 q} / \nu^{1+2 \theta}$ and $A^{\prime}$ $=D_{\rho} \Gamma(2 \theta) A / \nu^{1+2 \theta}$. We see that, in the rhs of Eq. (23), the terms $A_{2 q}^{\prime} L^{2(\chi-q)} r^{2 q}$ with $q<\chi$ are all dominant over the ordinary dynamic scaling term $A^{\prime} r^{2 \chi}$, since $L \gg r$. To compare them with Eq. (20), it is just like the time $t$ in the intermediate time asymptote of $G(r, t)$ being substituted by $O\left(L^{z} / \nu\right)$.

From the obtained early, intermediate, and late time asymptotes of $G(r, t)$, we have analytically shown that $G(r, t)$ of the growth processes described by Eqs. (3) and (4) with $2 m \theta+\rho+(2 m-1) / 2>1$ does satisfy, to the leading order, the anomalous dynamic scaling ansatz given in Eq. (2) with $l$ substituted by $r$. The local roughness exponent $\chi^{\prime}=1$ (independent of $m, \rho$, and $\theta$ ) for all the superrough growth processes described by Eqs. (3) and (4), while the exponent $\kappa=\chi-1=2 m \theta+\rho+(2 m-3) / 2$. In addition to the different spatial scaling behavior between $G(r, t)$ and the global interfacial width, the other key feature of anomalous dynamic scaling ansatz is that, at the regime $r^{z} / \nu \ll t \ll L^{z} / \nu, G(r, t)$ does not saturate but still increases with time and the lateral system size $L$ enters as an important cutoff for this superrough situation. In the thermodynamic limit $(L \rightarrow \infty), G(r, t)$ then can increase with time indefinitely. For illustration, Fig. 2 shows the numerical solution of $G(r, t)$ vs $t$ in the log-log scale for the interfaces described by Eqs. (3) and (4) with $m=1,2,3$, and the noise correlation indices $\rho=0.1, \theta=0.3$. Thus, the corresponding values of the scaling exponents are $\chi=1.2,2.8,4.4, z=2,4,6$, and $\kappa(=\chi-1)=0.2,1.8,3.4$ for the $m=1,2,3$ cases, respectively. The parameters are set to be $\nu=1, D_{\rho}=1, r=10$, and the lateral system size $L=10^{5}$ (for the $m=1$ case), 3000 (for $m=2$ ), 500 (for $m=3$ ). For visibility, the data points for the $m=2$ and 3 cases are shifted upward by 5 and 10 units, respectively, in Fig. 2. The straight lines with the slope equal to $2 \chi / z$ and $2 \kappa / z[=2(\chi-1) / z]$ are drawn along the data to make the crossover more visible.The figure clearly shows that $G(r, t)$ evolves through three different time regimes, distinct from the ordinary dynamic scaling behaviors. In addition, Fig. 3 shows the saturated value of the equal-time height difference correlation function, $G_{\text {sat }}(r, L)$, vs the lateral system size $L$ in the log-log scale for the growth processes described by Eqs. (3) and (4) with $m=2$, and the noise indices $(\rho, \theta)=(0.1,0.1),(0.2,0.3)$, and $(0.4,0.4)$. Thus, the corresponding values of the scaling exponents are $(\chi, z, \kappa)=(2,4,1), \quad(2.9,4,1.9), \quad$ and $(3.5,4,2.5)$ for the $(\rho, \theta)=(0.1,0.1),(0.2,0.3)$, and $(0.4,0.4)$ cases, respectively. The parameters are set to be $\nu=1, D_{\rho}$ $=1$, and $r=10$. The straight lines with the slope equal to $2 \kappa[=2(\chi-1)]$ are drawn along the data. All the data points fit the analytical prediction very well. Note that, for the ordinary dynamic scaling ansatz, the saturated value of $G(r, t)$ is independent of the lateral system size $L$, if $r \ll L$. In contrast, $G_{\text {sat }}(r, L) \rightarrow \infty$ as $L \rightarrow \infty$ for the superrough interfaces. In Sec. $\mathrm{V}$, we will discuss in detail this intriguing feature, local orientational instability, of the superrough interfaces.

\section{DIFFERENT-TIME HEIGHT DIFFERENCE CORRELATION FUNCTION AT THE STEADY STATE}

For completeness, we also study the different-time height difference correlation function at the steady state, which is defined as

$$
\left.G_{s}(r, \tau) \equiv \overline{\left\langle[h(x, t)-h(x+r, t+\tau)]^{2}\right\rangle_{L}}\right|_{t \gg L^{z / \nu}} .
$$

By performing some calculation, we have

$$
\begin{aligned}
\left.\overline{\langle h(x, t) h(x+r, t+\tau)\rangle_{L}}\right|_{t \gg L^{z / \nu}} \\
\simeq \frac{2 D_{\rho}}{L \nu} \sum_{n=1}^{\infty} \frac{e^{-i k_{n} r}}{k_{n}^{2 m+2 \rho}}\left(\int_{0}^{\infty} d \tau^{\prime}+e^{2 \nu k_{n}^{2 m} \tau} \int_{\tau}^{\infty} d \tau^{\prime}\right. \\
\left.\quad+\int_{0}^{\tau} d \tau^{\prime} e^{2 \nu k_{n}^{2 m} \tau^{\prime}}\right) e^{-\nu k_{n}^{2 m}\left(\tau+\tau^{\prime}\right)}\left(\tau^{\prime}\right)^{2 \theta-1},
\end{aligned}
$$

leading to

$$
\begin{aligned}
& G_{s}(r, \tau) \simeq \frac{2 D_{\rho}}{L \nu} \sum_{n=1}^{\infty} \frac{1}{k_{n}^{2 m+2 \rho}}\left[2 \int_{0}^{\infty} d \tau^{\prime}-\cos k_{n} r\left(e^{-\nu k_{n}^{2 m} \tau} \int_{0}^{\infty} d \tau^{\prime}\right.\right. \\
& \left.\left.+e^{\nu k_{n}^{2 m} \tau} \int_{\tau}^{\infty} d \tau^{\prime}+e^{-\nu k_{n}^{2 m} \tau} \int_{0}^{\tau} d \tau^{\prime} e^{2 \nu k_{n}^{2 m} \tau^{\prime}}\right)\right] \\
& \times e^{-\nu k_{n}^{2 m} \tau^{\prime}}\left(\tau^{\prime}\right)^{2 \theta-1} \text {. }
\end{aligned}
$$


We then derive the asymptote of $G_{s}(r, \tau)$ in the limit of $r^{z} / \nu \ll \tau \ll L^{z} / \nu$ as follows:

$$
\begin{aligned}
& G_{s}(r, \tau)-G_{s}(r, 0) \simeq \frac{D_{\rho}}{\pi \nu^{1+2 \theta}} \int_{0}^{\infty} \frac{d k \cos k r}{k^{2 m+2 \rho+4 m \theta}}\left(2 \int_{0}^{\infty} d \tau^{\prime}-e^{-\nu k^{2 m} \tau} \int_{0}^{\infty} d \tau^{\prime}-e^{\nu k^{2 m} \tau} \int_{\nu k^{2 m} \tau}^{\infty} d \tau^{\prime}-e^{-\nu k^{2 m} \tau} \int_{0}^{\nu k^{2 m} \tau} d \tau^{\prime} e^{2 \tau^{\prime}}\right) e^{-\tau^{\prime}}\left(\tau^{\prime}\right)^{2 \theta-1} \\
& =\frac{D_{\rho}}{\pi \nu^{1+2 \theta} k_{c}^{4 m \theta+2 \rho+2 m-1}} \int_{0}^{\infty} d \tau^{\prime} e^{-\tau^{\prime}}\left(\tau^{\prime}\right)^{2 \theta-1}\left(2 \int_{0}^{\infty} d x-\int_{0}^{\infty} d x e^{-x^{2 m}}-\int_{0}^{\left(\tau^{\prime}\right)^{1 / 2 m}} d x e^{x^{2 m}}-e^{2 \tau^{\prime}} \int_{\left(\tau^{\prime}\right)^{1 / 2 m}}^{\infty} d x e^{-x^{2 m}}\right) \\
& \times \frac{\cos k_{c} r x}{x^{4 m \theta+2 \rho+2 m}},
\end{aligned}
$$

with $k_{c} \equiv(\nu \tau)^{-1 / 2 m}$. By performing integration by parts systematically and using the following relations [19]:

$$
\begin{gathered}
\int_{0}^{\infty} x^{\alpha-1}\left\{\begin{array}{c}
\sin b x \\
\cos b x
\end{array}\right\} d x=\frac{\Gamma(\alpha)}{b^{\alpha}}\left\{\begin{array}{c}
\sin (\alpha \pi / 2) \\
\cos (\alpha \pi / 2)
\end{array}\right\} \text { with } b>0 \text { and }\left\{\begin{array}{c}
-1<\operatorname{Re} \alpha<1 \\
0<\operatorname{Re} \alpha<1
\end{array}\right\}, \\
\int_{0}^{\infty} x^{\alpha} e^{-x^{2 m}} d x=\frac{1}{2 m} \Gamma\left(\frac{\alpha+1}{2 m}\right),
\end{gathered}
$$

we can first obtain the following double integrals involved in the asymptote of $G_{s}(r, \tau)$ :

$$
\begin{aligned}
& \int_{0}^{\infty} d \tau^{\prime} e^{-\tau^{\prime}}\left(\tau^{\prime}\right)^{2 \theta-1} \int_{0}^{\infty} e^{-x^{2 m}} \frac{d x}{x^{\alpha}}\left\{\begin{array}{c}
\sin k_{c} r x \\
\cos k_{c} r x
\end{array}\right\}=\Gamma(2 \theta) \sum_{j=0}^{\infty} \frac{(-1)^{j}}{2 m(2 j+\mu) !}\left(k_{c} r\right)^{2 j+\mu} \Gamma\left(\frac{2 j+\mu-\alpha+1}{2 m}\right) \quad \text { with }\left\{\begin{array}{l}
\mu=1 \\
\mu=0
\end{array}\right\}, \\
& \int_{0}^{\infty} d \tau^{\prime} e^{-\tau^{\prime}}\left(\tau^{\prime}\right)^{2 \theta-1} \int_{0}^{\left(\tau^{\prime}\right)^{1 / 2 m}} e^{x^{2 m}} \frac{d x}{x^{\alpha}}\left\{\begin{array}{c}
\sin k_{c} r x \\
\cos k_{c} r x
\end{array}\right\}=\sum_{j=0}^{\infty} \frac{(-1)^{j}}{2 m(2 j+\mu) !} \frac{\Gamma\left(\frac{2 j+\mu-\alpha+1}{2 m}\right) \Gamma\left(1-2 \theta-\frac{2 j+\mu-\alpha+1}{2 m}\right)}{\Gamma(1-2 \theta)}\left(k_{c} r\right)^{2 j+\mu} \\
& \text { with }\left\{\begin{array}{l}
\mu=1 \\
\mu=0
\end{array}\right\} \\
& \int_{0}^{\infty} d \tau^{\prime} e^{\tau^{\prime}}\left(\tau^{\prime}\right)^{2 \theta-1} \int_{\left(\tau^{\prime}\right)^{1 / 2 m}}^{\infty} e^{-x^{2 m}} \frac{d x}{x^{\alpha}}\left\{\begin{array}{c}
\sin k_{c} r x \\
\cos k_{c} r x
\end{array}\right\}=\sum_{j=0}^{\infty} \frac{(-1)^{j}}{2 m(2 j+\mu) !} \frac{\Gamma(2 \theta) \Gamma\left(1-2 \theta-\frac{2 j+\mu-\alpha+1}{2 m}\right)}{\Gamma\left(1-\frac{2 j+\mu-\alpha+1}{2 m}\right)}\left(k_{c} r\right)^{2 j+\mu} \text { with }\left\{\begin{array}{l}
\mu=1 \\
\mu=0
\end{array}\right\} .
\end{aligned}
$$

By employing the earlier obtained relations and with some tedious calculation, we eventually obtain the asymptote of $G_{s}(r, \tau)$ as follows:

$$
\left.G_{s}(r, \tau)\right|_{r^{z / \nu} \tau \ll L^{z / \nu}}-G_{S}(r, 0) \simeq \frac{D_{\rho} k_{c}^{1-2 m-2 \rho-4 m \theta}}{\pi \nu^{1+2 \theta}}\left[\sum_{q=1}^{\infty} d_{2 q}\left(k_{c} r\right)^{2 q}+d\left(k_{c} r\right)^{4 m \theta+2 \rho+2 m-1}\right]
$$

with the coefficients

$$
\begin{aligned}
d_{2 q}= & \frac{(-1)^{q}}{(2 q) !(2 m-1+2 \rho+4 m \theta-2 q)}\left[\Gamma(2 \theta) \Gamma\left(\frac{1+2 q-2 \rho}{2 m}-2 \theta\right)\right. \\
& \left.-\frac{\Gamma\left(\frac{1+2 q-2 \rho}{2 m}-2 \theta\right) \Gamma\left(1-\frac{1+2 q-2 \rho}{2 m}\right)}{\Gamma(1-2 \theta)}+\frac{\Gamma(2 \theta) \Gamma\left(1-\frac{1+2 q-2 \rho}{2 m}\right)}{\Gamma\left(1+2 \theta-\frac{1+2 q-2 \rho}{2 m}\right)}\right]
\end{aligned}
$$

for $q<m$, 


$$
\begin{aligned}
d_{2 q}= & \frac{(-1)^{q}}{(2 m-1+2 \rho+4 m \theta-2 q)}\left[\frac{1}{(2 q) !}-\frac{\Gamma(2 \rho+4 m \theta+1)}{\Gamma(2 m+2 \rho+4 m \theta)(2 q-2 m+1) !}\right]\left[\Gamma(2 \theta) \Gamma\left(\frac{1+2 q-2 \rho}{2 m}-2 \theta\right)\right. \\
& \left.-\frac{\Gamma\left(\frac{1+2 q-2 \rho}{2 m}-2 \theta\right) \Gamma\left(1-\frac{1+2 q-2 \rho}{2 m}\right)}{\Gamma(1-2 \theta)}+\frac{\Gamma(2 \theta) \Gamma\left(1-\frac{1+2 q-2 \rho}{2 m}\right)}{\Gamma\left(1+2 \theta-\frac{1+2 q-2 \rho}{2 m}\right)}\right]-(-1)^{q}\left[\Gamma(2 \theta) \Gamma\left(\frac{1+2 q-2 \rho}{2 m}-1-2 \theta\right)\right. \\
& \left.-\frac{\Gamma\left(\frac{1+2 q-2 \rho}{2 m}-1-2 \theta\right) \Gamma\left(2-\frac{1+2 q-2 \rho}{2 m}\right)}{\Gamma(1-2 \theta)}+\frac{\Gamma(2 \theta) \Gamma\left(2-\frac{1+2 q-2 \rho}{2 m}\right)}{\Gamma\left(2+2 \theta-\frac{1+2 q-2 \rho}{2 m}\right)}\right]
\end{aligned}
$$

for $q \geqslant m$, and

$$
\begin{aligned}
d= & (-1)^{m+1} \frac{4 m \Gamma(2 \rho+4 m \theta+1)}{\Gamma(2 m+2 \rho+4 m \theta)} \Gamma(2 \theta) \Gamma(-2 \rho-4 m \theta) \\
& \times \sin [(\rho+2 m \theta) \pi] .
\end{aligned}
$$

After substituting $k_{c}=(\nu \tau)^{-1 / 2 m}$, the global roughness exponent $\chi=2 m \theta+\rho+(2 m-1) / 2$, and the dynamic exponent $z$ $=2 m$ into Eq. (28), the asymptote of $G_{s}(r, \tau)$ can also be written as

$$
\left.G_{S}(r, \tau)\right|_{r^{z} / \nu \ll \tau \ll L / \nu}-G_{S}(r, 0) \simeq \sum_{q=1}^{\infty} d_{2 q}^{\prime} \tau^{2(\chi-q) / z} r^{2 q}+d^{\prime} r^{2 \chi}
$$

with the coefficients $d_{2 q}^{\prime}=d_{2 q}\left[2 D_{\rho} \nu^{2(\chi-q) / z} /\left(\pi \nu^{1+2 \theta}\right)\right]$ and $d^{\prime}$ $=d\left[2 D_{\rho} /\left(\pi \nu^{1+2 \theta}\right)\right]$. Indeed, we see that the terms $d_{2 q}^{\prime} \tau^{2(\chi-q) / z} r^{2 q}$ with $q<\chi$ are all dominant over the ordinary dynamic scaling term $d^{\prime} r^{2 \chi}$ in the limit of $\tau \gg r^{z} / \nu$.

\section{LOCAL WIDTH AND MACROSCOPIC STRUCTURE FORMATION}

With the extensive studies about the asymptotes of $G(r, t)$, one can easily obtain the asymptotes of the local interfacial width $w(l, t)$, defined as

$$
w^{2}(l, t) \equiv \overline{\left\langle\left\langle\left(h(x, t)-\langle h(x, t)\rangle_{l}\right)^{2}\right\rangle_{l}\right\rangle_{L}}
$$

with $\langle\ldots\rangle_{l}$ denoting the lateral spatial average calculated within a local window of lateral size $l$ throughout this paper. From the definitions of $G(r, t)$ and $w(l, t)$, the relation between $w(l, t)$ and $G(r, t)$ is obtained

$$
w^{2}(l, t)=\frac{1}{l^{2}} \int_{0}^{l}(l-r) G(r, t) d r .
$$

Then, following the derivation in Sec. III, we obtain the asymptotes of the local interfacial width $w(l, t)$ as follows:

(i) For $t \ll l / \nu$ :

$$
w^{2}(l, t) \sim O\left(\frac{D_{\rho}}{\nu^{(1-2 \rho) / z}} t^{2 \chi / z}\right)
$$

(ii) For $l^{z} / \nu \ll t \ll L^{z} / \nu$ :

$$
w^{2}(l, t) \simeq \sum_{p=1}^{\infty} C_{2 p}^{\prime \prime} t^{2(\chi-p) / z} l^{2 p}+C^{\prime \prime} l^{2 \chi}
$$

with the coefficients $C_{2 p}^{\prime \prime}=C_{2 p}^{\prime} /[(2 p+1)(2 p+2)]$ and $C^{\prime \prime}$ $=C^{\prime} /[(2 \chi+1)(2 \chi+2)]$.

(iii) For $t \gg L^{z} / \nu$ :

$$
w^{2}(l, t) \simeq \sum_{p=1}^{\infty} A_{2 p}^{\prime \prime} L^{2(\chi-p)} l^{2 p}+A^{\prime \prime} l^{2 \chi}
$$

with the coefficients $A_{2 p}^{\prime \prime}=A_{2 p}^{\prime} /[(2 p+1)(2 p+2)]$ and $A^{\prime \prime}$ $=A^{\prime} /[(2 \chi+1)(2 \chi+2)]$. Note that, in the intermediate time regime $l^{z} / \nu \ll t \ll L^{z} / \nu$, the leading anomalous term $\left[C_{2}^{\prime \prime} t^{2(\chi-1) / z} l^{2}\right]$ and the subleading anomalous terms $\left\{C_{4}^{\prime \prime} t^{2(\chi-2) / z} l^{4}, \ldots, C_{2[\chi]}^{\prime \prime} t^{2(\chi-[\chi]) / z} l^{2[\chi]}\right\}$ dominate over the ordinary dynamic scaling term $C^{\prime \prime} l^{2 \chi}$ and, in the late time regime $t \gg L^{z} / \nu$, the leading anomalous term $\left[A_{2}^{\prime \prime} L^{2(\chi-1)} l^{2}\right]$ and the subleading anomalous terms $\left\{A_{4}^{\prime \prime} L^{2(\chi-2)} l^{4}, \ldots, A_{2[\chi]}^{\prime \prime} L^{2(\chi-[\chi])} l^{2[\chi]}\right\}$ dominate over the ordinary dynamic scaling term $A^{\prime \prime} l^{2 \chi}$ for $w^{2}(l, t)$ of the growth processes described by Eqs. (3) and (4) with $2 m \theta+\rho+(2 m$ $-1) / 2>1$. The main reason causing the difference between the local and global scaling is that all these superrough interfaces are associated with local orientational instability but, at the same time, with periodic boundary conditions restricting the development of global interfacial widths, as illustrated in Fig. 1. 
Finally, we would like to quantitatively investigate the temporal development of local orientational instability of this class of superrough growth processes. The term "local interfacial orientation" quantitatively refers to $s(l, t)$, the slope of a straight line segment obtained by least squares fit to the interfacial configuration in the local window of size $l$ at a given time $t$. By some calculation, we have

$$
s(l, t)=\frac{12}{l^{2}}\left\langle\left(x-\langle x\rangle_{l}\right) h(x, t)\right\rangle_{l} .
$$

Subsequently, after some tedious calculation, we obtain the explicit relation between $G(r, t)$ and $\overline{\left\langle s^{2}(l, t)\right\rangle_{L}}{ }^{1 / 2}$, the average magnitude of the local interfacial orientation, as follows:

$$
\overline{\left\langle s^{2}(l, t)\right\rangle_{L}}=\frac{12}{l^{6}} \int_{0}^{l} G(r, t)\left(3 l^{2} r-2 r^{3}-l^{3}\right) d r .
$$

Next, following the derivation in Sec. III, we obtain the asymptotes of $\overline{\left\langle s^{2}(l, t)\right\rangle_{L}}$ as follows:

(i) For $t \ll l^{z} / \nu$ :

$$
\overline{\left\langle s^{2}(l, t)\right\rangle_{L}} \sim O\left(D_{\rho} t^{1+2 \theta} l^{2 \rho-3}\right) .
$$

(ii) For $l^{z} / \nu \ll t \ll L^{z} / \nu$ :

$$
\overline{\left\langle s^{2}(l, t)\right\rangle_{L}} \simeq \sum_{p=1}^{\infty} \widetilde{C}_{2 p} t^{2(\chi-p) / z} l^{2 p-2}+\tilde{C} l^{2 \chi-2}
$$

with the coefficients $\widetilde{C}_{2 p}=\{18 p /[(p+1)(p+2)(2 p+1)]\} C_{2 p}^{\prime}$ and $\widetilde{C}=\{18 \chi /[(\chi+1)(\chi+2)(2 \chi+1)]\} C^{\prime}$.

(iii) For $t \gg L^{z} / \nu$ :

$$
\overline{\left\langle s^{2}(l, t)\right\rangle_{L}} \simeq \sum_{p=1}^{\infty} \widetilde{A}_{2 p} L^{2(\chi-p)} l^{2 p-2}+\widetilde{A} l^{2 \chi-2}
$$

with the coefficients $\widetilde{A}_{2 p}=\{18 p /[(p+1)(p+2)(2 p+1)]\} A_{2 p}^{\prime}$ and $\tilde{A}=\{18 \chi /[(\chi+1)(\chi+2)(2 \chi+1)]\} A^{\prime}$.

Recall that, for the systems obeying the ordinary dynamic scaling ansatz, the local interfacial orientation saturates quickly after the growth time reaching the regime $t \gg l^{z} / \nu$. In contrast, for the growth processes described by Eqs. (3) and (4) with $2 m \theta+\rho+(2 m-1) / 2>1$, the leading anomalous term $\left[\widetilde{C}_{2} t^{2(x-1) / z}\right]$ and the subleading anomalous terms $\left\{\widetilde{C}_{4} t^{2(x-2) / z} l^{2}, \ldots, \widetilde{C}_{2[\chi]} t^{2(x-[\chi]) / z} l^{2[\chi]-2}\right\}$ dominate over the ordinary dynamic scaling term $\widetilde{C} l^{2 \chi-2}$ in the intermediate time regime $l^{z} / \nu \ll t \ll L^{z} / \nu$ and the system size $L$ enters as an important cutoff for the temporal development of local orientational instability.

\section{CONCLUSION}

In conclusion, we take an extensive study of a class of superrough interfacial growth processes with finite lateral system size $L$ in $1+1$ dimensions described by linear growth equations with spatiotemporally power-law decaying correlated noise, Eqs. (3) and (4). By using the scaling analysis, we have the global roughness exponent $\chi=2 m \theta+\rho+(2 m$ $-1) / 2$ and the dynamic exponent $z=2 m$, independent of $\rho$ and $\theta$. Thus, the interfacial growth processes described by Eqs. (3) and (4) with $2 m \theta+\rho+(2 m-1) / 2>1$ display superroughening phenomena. Since some of these processes have extremely long relaxation time, we first develop a very efficient method capable of simulating the interface morphology of these growth processes even in the very late time. We numerically observe that this class of superrough growth processes indeed gradually develop macroscopic structures with the lateral size comparable to the lateral system size. The cause of the anomalous dynamic scaling behaviors in the superrough growth processes governed by Eqs. (3) and (4) is totally attributed to the formation of global mountains and valleys in the interface morphology. The asymptotes of the equal-time height difference correlation function $G(r, t)$ are summarized as follows:

$$
\left\{\begin{array}{l}
G(r, t) \sim O\left(\frac{D_{\rho}}{\nu^{(1-2 \rho) / z}} t^{2 \chi / z}\right) \quad \text { for } t \ll r^{z} / \nu ; \\
G(r, t) \simeq \sum_{q=1}^{\infty} C_{2 q^{\prime} t^{2(\chi-q) / z} r^{2 q}+C^{\prime} r^{2 \chi} \quad \text { for } r^{z} / \nu \ll t \ll L^{z} / \nu ;}^{\infty} \\
G(r, t) \simeq \sum_{q=1}^{\infty} A_{2 q}^{\prime} L^{2(\chi-q)} r^{2 q}+A^{\prime} r^{2 \chi} \quad \text { for } t \gg L^{z} / \nu .
\end{array}\right.
$$

Note that, in the intermediate time regime, the terms $\left\{C_{2}^{\prime} t^{2(x-1) / z} r^{2}, \ldots, C_{2[\chi]^{\prime}}^{\prime} t^{2(\chi-[\chi]) / z} r^{2[x]}\right\}$ are all dominant over the ordinary dynamic scaling term $C^{\prime} r^{2 \chi}$ and, in the late time regime, the terms $\left\{A_{2}^{\prime} L^{2(\chi-1)} r^{2}, \ldots, A_{2[\chi}^{\prime} L^{2(\chi-[\chi])} r^{2[\chi]}\right\}$ are all dominant over the ordinary dynamic scaling term $A^{\prime} r^{2 \chi}$ for the growth processes described by Eqs. (3) and (4) with $2 m \theta+\rho+(2 m-1) / 2>1$. The local interfacial width has the asymptotic behaviors similar to those of $G(r, t)$ and distinct from the scaling behaviors of the global interfacial width. Through our detailed analytic study, we explicitly obtain not only the leading anomalous dynamic scaling term but also all the subleading anomalous dynamic scaling terms which are dominant over the ordinary dynamic scaling term. Next, we take a quantitative study on the temporal development of local orientational instability of these superrough interfaces. The asymptotes of the average magnitude of local interfacial orientation, $\overline{\left\langle s^{2}(l, t)\right\rangle_{L}}{ }^{1 / 2}$, are summarized as follows: 


$$
\begin{cases}\overline{\left\langle s^{2}(l, t)\right\rangle_{L}} \sim O\left(D_{\rho} t^{1+2 \theta} l^{2 \rho-3}\right), & \text { for } t \ll l^{z} / \nu ; \\ \overline{\left\langle s^{2}(l, t)\right\rangle_{L}} \simeq \sum_{p=1}^{\infty} \widetilde{C}_{2 p} t^{2(\chi-p) / z} l^{2 p-2}+\widetilde{C} l^{2 \chi-2}, & \text { for } l^{z} / \nu \ll t \ll L^{z} / \nu \\ \overline{\left\langle s^{2}(l, t)\right\rangle_{L}} \simeq \sum_{p=1}^{\infty} \widetilde{A}_{2 p} L^{2(\chi-p)} l^{2 p-2}+\widetilde{A} l^{2 \chi-2}, & \text { for } t \gg L^{z} / \nu .\end{cases}
$$

The term "local orientational instability" quantitatively refers to the anomalous terms $\left.\left\{\widetilde{C}_{2} t^{2(x-1) / z}, \ldots, \widetilde{C}_{2[\chi \chi}\right]^{2(x-[\chi]) / z} l^{2[\chi]-2}\right\}$ dominant over the ordinary dynamic scaling term $\widetilde{C} l^{2 x-2}$ in the intermediate time regime $l^{z} / \nu \ll t \ll L^{z} / \nu$ and the global lateral system size $L$ enters as an important cutoff due to the periodic boundary conditions.

By contrast, the well-known Kardar-Parisi-Zhang (KPZ) equation [20] with spatiotemporally correlated noise in 1 +1 dimensions do obey the ordinary dynamic scaling ansatz $[21,22]$. Especially for the case of purely spatially correlated noise $(\rho \neq 0$ and $\theta=0)$, the $(1+1)$-dimensional KPZ interface conserves the Galilean invariance and thus holds the scaling relation $\chi+z=2$, very different from the superrough interfaces described by Eqs. (3) and (4). The explicit relation between the scaling exponents $(\chi$ and $z)$ and the noise correlation indices $(\rho$ and $\theta)$ for the $(1+1)$-dimensional KPZ interfaces with purely spatially (or temporally) correlated noise has been obtained by the extensive dynamical renormalization-group calculations in Refs. [21,22]. For explicit comparison with the superrough interfaces described by Eqs. (3) and (4), we list out their results as follows:

(i) for $\rho \neq 0$ and $\theta=0$ :

$$
\left\{\begin{array}{l}
\chi=1 / 2 \text { and } z=3 / 2 \quad \text { with } 0<\rho \leqslant 1 / 4, \\
\chi=\frac{1+2 \rho}{3} \text { and } z=\frac{5-2 \rho}{3} \quad \text { with } 1 / 4<\rho<1
\end{array}\right.
$$

(ii) for $\rho=0$ and $\theta \neq 0$ :

$$
\left\{\begin{array}{l}
\chi=1 / 2 \text { and } z=3 / 2 \quad \text { with } 0<\theta \leqslant 0.167, \\
\chi \simeq 1.69 \theta+0.22 \text { and } z \simeq \frac{1.44+3.38 \theta}{1+2 \theta} \\
\text { with } 0.167<\theta<1 / 2 .
\end{array}\right.
$$

The most distinct difference between the KPZ interfaces and the superrough interfaces described by Eqs. (3) and (4) is that in the former cases there exists a critical value of the noise correlation index $\left(\rho_{c}=1 / 4\right.$ or $\left.\theta_{c} \simeq 0.167\right)$ marking the stability boundary between the short-range and long-range noise fixed points, while for the latter cases the long-range noise correlations are always prevalent in the renormalization group sense. In a word, the presence of correlations in noise can change the scaling exponents and produce a family of continuously changing universality classes. This might be one of the reasons why the values of the scaling exponents reported in experiments scatter over a considerable range. The results derived in this report can help for pinning down this issue and offer a reference for the interpretation of experiments.

\section{ACKNOWLEDGMENTS}

The work of N.-N.P. is supported in part by the National Science Council of the Republic of China under Grant No. NSC 92-2112-M-002-017. The work of W.-J.T. is supported in part by the National Science Council of the Republic of China under Grant No. NSC 92-2112-M-032-011.
[1] A.-L. Barabasi and H. E. Stanley, Fractal Concepts in Surface Growth (Cambridge University Press, New York, 1995); T. Halpin-Healy and Y.-C. Zhang, Phys. Rep. 254, 215 (1995); P. Meakin, Fractals, Scaling \& Growth Far from Equilibrium (Cambridge University Press, Cambridge, 1998); J. Krug and H. Spohn, in Solids Far from Equilibrium: Growth, Morphology, and Defects, edited by C. Godreche (Cambridge University Press, New York, 1990).

[2] F. Family and T. Vicsek, J. Phys. A 18, L75 (1985).

[3] H.-N. Yang, Y.-P. Zhao, G.-C. Wang, and T.-M. Lu, Phys. Rev. Lett. 76, 3774 (1996).

[4] J. H. Jeffries, J. K. Zuo, and M. M. Craig, Phys. Rev. Lett. 76, 4931 (1996).

[5] S. Morel, J. Schmittbuhl, J. M. López, and G. Valentin, Phys. Rev. E 58, 6999 (1998).
[6] M. Schroeder, M. Siegert, D. E. Wolf, J. D. Shore, and M. Plischke, Europhys. Lett. 24, 563 (1993).

[7] S. Das Sarma, C. J. Lanczycki, R. Kotlyar, and S. V. Ghaisas, Phys. Rev. E 53, 359 (1996).

[8] N.-N. Pang and W.-J. Tzeng, Phys. Rev. E 61, 3212 (2000).

[9] E. Koscielny-Bunde et al., Phys. Rev. Lett. 81, 729 (1998).

[10] M. Ausloos and K. Ivanova, Eur. Phys. J. B 20, 537 (2001).

[11] A. Bunde et al., Phys. Rev. Lett. 85, 3736 (2000).

[12] S. V. Buldyrev et al., Phys. Rev. E 51, 5084 (1995).

[13] M. Cattani and M. C. Salvadori, Thin Solid Films 376, 264 (2000).

[14] A. Király and I. M. Jánosi, Phys. Rev. E 65, 051102 (2002).

[15] D. Lee, N. L. Port, W. Kruse, and A. P. Georgopoulos, J. Neurosci. 18, 1161 (1998).

[16] D. J. DeShazer, J. García-Ojalvo, and R. Roy, Phys. Rev. E 
67, 036602 (2003).

[17] S. F. Edwards and D. R. Wilkinson, Proc. R. Soc. London, Ser. A 381, 17 (1982).

[18] D. E. Wolf and J. Villain, Europhys. Lett. 13, 389 (1990).

[19] See, for example, An Atlas of Functions, edited by J. Spanier and K. B. Oldham (Springer-Verlag, Berlin, 1987); Integrals and Series, edited by A. P. Prudnikov, Y. A. Brychkov, and O.
I. Marichev (Gordon and Breach, New York, 1986).

[20] M. Kardar, G. Parisi, and Y.-C. Zhang, Phys. Rev. Lett. 56, 889 (1986).

[21] E. Medina, T. Hwa, M. Kardar, and Y.-C. Zhang, Phys. Rev. A 39, 3053 (1989).

[22] H. K. Janssen, U. C. Täuber, and E. Frey, Eur. Phys. J. B 9, 491 (1999). 